\title{
Auswahlaxiom in der Algebra
}

\section{Doctoral Thesis}

\section{Author(s):}

Läuchli, Hans

Publication date:

1962

Permanent link:

https://doi.org/10.3929/ethz-a-000087895

Rights / license:

In Copyright - Non-Commercial Use Permitted 
Prom. Nr. 3179

\title{
Answahlaxiom in der Algebra
}

\author{
VON DER \\ EIDGENÖSSISCHEN TECHNISCHEN HOCHSCHULE IN ZƯRICH \\ ZUR ERLANGUNG DER WÜRDE EINES DOKTORS DER MATHEMATIK
}

GENEHMIGTE

\section{PROMOTIONSARBEIT}

\author{
VORGELEGT VON \\ HANS LĀUCHLI \\ VON AARAU \\ DIPL. MATH. ETH \\ Referent: Herr Prof. Dr. E. SpeCker \\ Korreferent: Herr Prof. Dr. B. EckmanN
}

1962

ART. INSTITUT ORELL FÜSSI AG, ZURICH 


\title{
Auswahlaxiom in der Algebra
}

\author{
von H. Lä̈OHLr, Winterthur
}

\section{Einleitung}

In der vorliegenden Arbeit wird von einigen mathematischen Sätzen, vorwiegend von Sätzen aus der Algebra, gezeigt, daß sie nicht ohne Auswahlaxiom beweisbar sind.

$\mathrm{Zu}$ diesem Zwecke werden Modelle für die Mengenlehre konstruiert nach einer Methode, wie sie erstmals von A. Fraknker in [3], später von A. MosTOWSKI [6] und R. FraIssí [4] angewendet wurde. E. SPECKER beschrieb diese Methode in ihrer allgemeinsten Art in [7].

Wir geben in Kapitel I im wesentlichen eine Zusammenfassung dieser Beschreibung. Als Rahmen dient das von P. Bernays [1] aufgestellte Axiomensystem für die Mengenlehre. Die Kapitel II-VI bringen Anwendungen der in Kapitel I beschriebenen Methode.

Wir zeigen in Kapitel II, daß die folgenden Sätze aus der Körpertheorie nicht ohne Auswahlaxiom beweisbar sind: Jeder Körper besitzt einen algebraisch-abgeschlossenen Erweiterungskörper; jeder formal-reelle Körper besitzt einen reell-abgeschlossenen Erweiterungskörper; jeder algebraisch-abgeschlossene, algebraische Erweiterungskörper eines abzählbaren Körpers ist abzählbar (insbesondere: jeder algebraische Erweiterungskörper des Körpers der rationalen Zahlen ist abzählbar); zwischen einem formal-reellen Körper und einer algebraisch-abgeschlossenen Erweiterung dieses Körpers gibt es einen reell-abgeschlossenen Körper.

In Kapitel III wird ein Modell konstruiert, worin ein (nicht endlich dimensionaler) Vektorraum $v$ existiert mit den folgenden Eigenschaften: a) Jeder echte Unterraum von $v$ ist endlich dimensional. Daraus ergibt sich beispielsweise, daß $v$ keine Basis (im algebraischen Sinne) besitzt, und daß der duale Raum $v^{*}$ nur aus der 0-Abbildung besteht. b) Die einzige zulässige Vektorraumtopologie auf $v$ ist die triviale (gröbste). c) Die einzigen linearen Selbstabbildungen von $v$ sind die Vielfachen der Identität.

In Kapitel IV wird gezeigt, daß der Schreiersche Satz über die Untergruppen freier Gruppen nicht ohne Auswahlaxiom beweisbar ist.

In Kapitel V wird ein Modell angegeben, worin isomorphe Vektorräume mit ungleich mächtigen Basen existieren. Das heißt der Satz, wonach alle Basen eines Vektorraumes gleich mächtig sind, ist nicht ohne Auswahlaxiom beweisbar. Im gleichen Modell existieren verschieden mächtige Mengen mit gleich 
mächtigen Potenzmengen. Nach derselben «Methode der direkten Summe», wie sie hier zur Anwendung gelangt, können viele weitere Beispiele behandelt werden.

In Kapitel VI wird im wesentlichen gezeigt, daß der Satz von Urysohn (aus der Topologie) nicht ohne Auswahlaxiom beweisbar ist. Dabei gelangt ein Modell zur Anwendung, das sich von allen vorher konstruierten insofern unterscheidet, als es kein Normalmodell ist (vgl. 1.6).

Da das Hauptgewicht der Arbeit auf Anwendungen der in [7] beschriebenen Methode zur Konstruktion von Modellen liegt und nicht auf axiomatischen Untersuchungen der Mengenlehre, wurde auf einen formalen Aufbau im Sinne der Mathematischen Logik verzichtet.

\section{Permutationsmodelle ${ }^{1}$ )}

1. Wir legen der Mengenlehre die Axiome I-VI des Axiomensystems von Bernays [1] zugrunde: Dies sind die Axiome der Extensionalität, der direkten Mengenkonstruktion, der Klassenkonstruktion, das Auswahlaxiom, das Axiom über die Darstellung von Klassen durch Mengen, das Unendlichkeitsaxiom.

Zusätzlich erfülle die Mengenlehre ein «Fundierungsaxiom bezüglich einer Basismenge $a_{0}{ }^{\text {». }}$

Das heißt a) Die Klasse $A$ derjenigen Mengen, die sich selbst als einziges Element enthalten, $A=\{x \mid x=(x)\}$, werde durch eine Menge repräsentiert: Die Basismenge $a_{0}$.

b) Jede nicht leere Klasse $C$ enthalte ein Element $c$, so daß $C \cap c \subset a_{0}$.

Dieses Axiom hat die folgende Bedeutung: Sei $\psi$ die Funktion, die jedem Paar $a, \alpha$, gebildet aus einer beliebigen Menge $a$ und einer Ordnungszahl $\alpha$, eine Menge $\psi(a, \alpha)$ zuordnet, so da $\beta$ gilt: $\psi(a, 0)=0, \psi(a, \alpha+1)$ ist die Potenzmenge von $\psi(a, \alpha)$, für eine Limeszahl $\lambda$ ist $\psi(a, \lambda)$ die Vereinigung der Mengen $\psi(a, \xi)$ mit $\xi \in \lambda . \Pi(a)$ sei die Vereinigungsklasse der Menge $\psi(a, \alpha)$, wo $\alpha$ die Klasse aller Ordnungszahlen durchläuft. Das Fundierungsaxiom bezüglich $a_{0}$ ist nun äquivalent mit der Forderung, $\operatorname{da} ß \Pi\left(a_{0}\right)$ die Allklasse sei.

Die Elemente der Basismenge $a_{0}$ übernehmen die Rolle der Urelemente in Zermelos Terminologie. Das klassische Fundierungsaxiom besagt, daß $\Pi(0)$ die Allklasse sei.

Das Fundierungsaxiom bezüglich $a_{0}$ ist konsistent mit den Axiomen I-VI; dabei darf die Mächtigkeit von $a_{0}$ frei vorgeschrieben werden (vgl. [7]).

Die von uns betrachtete Mengenlehre soll von abzählbarer Basis sein. $a_{0}$ wird zugleich Basismenge der Modelle sein, die wir, ausgehend von dieser

1) Vgl. mit der in [7], S. 193-199, gegebenen Darstellung. 